\title{
ON THE DEVELOPMENT OF A SCATTEROMETER-BASED CORRECTION FOR NWP WIND FORCING SYSTEMATIC ERRORS: IMPACT OF SATELLITE SAMPLING
}

\author{
Ana Trindade ${ }^{1}$, Marcos Portabella ${ }^{1}$,Wenming Lin ${ }^{1}$, Ad Stoffelen ${ }^{2}$ \\ ${ }^{1}$ Institut de Ciències del Mar (ICM-CSIC), Barcelona, Spain \\ ${ }^{2}$ Royal Netherlands Meteorological Institute (KNMI), De Bilt, The Netherlands
}

\begin{abstract}
Local systematic differences between scatterometer and global numerical weather prediction (NWP) model stress equivalent winds (SEW) are due to unresolved geophysical processes by the model, e.g., ocean currents and moist convection. A scatterometer-based correction, which contains the mesoscale information present in the Advanced Scatterometer (ASCAT) observations, sets the grounds for a high-resolution ocean forcing product. To assess the effectiveness of such correction, a Monte Carlo simulation procedure is applied to NWP SEW. It allows for a thorough evaluation of the NWP error reduction, which depends on the scatterometer sampling. The local NWP biases are reduced at the cost of a somewhat increased variance, and the total error mitigation is constrained to regions covered by the scatterometer at least 3 times over 5 days. Despite the limited sampling in the tropics, the real NWP corrected SEW over the West African coast show areas of increased wind variability associated to moist convection.
\end{abstract}

Index Terms - NWP, stress equivalent winds, systematic errors, mesoscale, West Africa

\section{INTRODUCTION}

There is an increasing demand for high spatial and temporal resolution gridded ocean vector winds in earth science fields such as, Meteorology, Oceanography and Climate [1]. To the current date, in what concerns ocean forcing, numerical weather prediction (NWP) model output is still widely used due to its high frequency sampling. However, such forcing products cannot properly resolve small-scale processes, like mesoscale eddies, ocean currents, air-sea fluxes, moist convection or coastal interaction. As shown in Figure 1, this leads to significant NWP wind local biases [2].

A scatterometer-based correction, using accurate, unbiased, high spatial resolution ocean vector winds from the Advanced Scatterometers (ASCATs) on board Metop satellites, is proposed as a new angle to tackle this problem, i.e., to reduce NWP local wind biases accounting for satellite sampling characteristics (note that the scatterometer temporal sampling is latitudinal dependent and rather poor in the

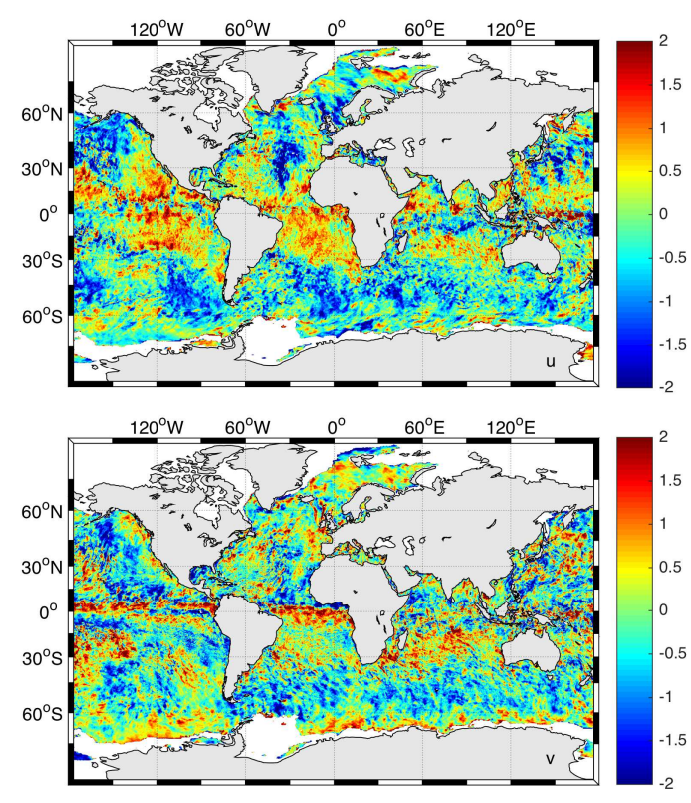

Fig. 1. Scatterometer correction for a given day, e.g., 14th April 2012. Differences between ASCAT-A (12.5 km) and ECMWF stress equivalent zonal $(\mathrm{u})$ and meridional $(\mathrm{v})$ wind components, accumulated over a 5-day time window (period from January $12^{\text {th }}$ to $\left.16^{\text {th }}, 2013\right)$. The colors represent the differences in $\mathrm{m} / \mathrm{s}$ (see color scale).

tropics). The correction consists of geolocated (i.e., at every ocean grid point), temporally-averaged differences between the scatterometer and the collocated NWP stress equivalent winds (SEW), i.e., 10-m equivalent neutral winds accounting for air density variations [3]. Since NWP local biases are relatively persistent at weekly scales (not shown) and in order to account for satellite sampling limitations, an optimal window of 5-days (i.e., the quasi-repeat cycle of Metop) was used to compute these differences.

This approach aims at correcting wind vector biases, but by performing such corrections at scatterometer grid resolution, additional wind vector variability is also introduced, which can be of added value in areas of, e.g., moist convec- 
tion.

The work is structure as follows. In Section 2, the data used in this study are briefly presented. In section 3, Monte Carlo simulations, are carried out to assess the limitations of using the above described method to introduce small-scales into NWP model winds. In particular, the effect of using one or two scatterometers to compute the correction, mimicking the temporal and spatial coverage of ASCAT-A and B, will be analyzed. In the nominal configuration (i.e., one scatterometer), the correction leads to improvement (i.e., effective reduction of NWP total error) only at high latitudes, where the scatterometer sampling is the largest. Accordingly, in the tropical band, where air-sea interaction is a crucial process, the correction shows the worst performance. In Section 4.2, a real case study encompassing the West African coast is further explored to assess the potential benefits of the proposed correction over poorly (scatterometer) sampled areas.

\section{DATA}

In order to provide a realistic simulation, NWP model forecast data are used as reference or truth. The dataset used consists of 1 month (January 2013), and several sets of five consecutive days ( $12^{\text {th }}$ to the $16^{\text {th }}$ of January, June, September and December 2013) of European Centre for Medium Range Weather Forecasts (ECMWF) hourly wind forecasts available from the ECMWF Meteorological Archival and Retrieval System (MARS). These model winds are then converted to SEW using a stand-alone version of the ECMWF surface layer model.

For the same periods, the scatterometer sampling is extracted from the Ocean \& Sea Ice Satellite Application Facility (OSI-SAF) 12.5-km Level 2 (L2) ASCAT-A and ASCATB BUFR products. Also note that real L2 ASCAT-A wind data is used to compute the NWP SEW-corrected fields in Section 4

\section{METHODOLOGY}

The Monte Carlo scheme is applied to the NWP SEW vector components, $\mathrm{u}$ and $\mathrm{v}$. As already mentioned, the scheme assumes ECMWF winds as the true wind field. This is then perturbed with different random and systematic error values to produce synthetic scatterometer, ASCAT, and model, NWP, winds. ASCAT is assumed unbiased and the total error standard deviation (SD) values (i.e., the square root of the sum of the systematic and the random error variances) for NWP and ASCAT (at scatterometer scales) are adopted from the literature [4] to be $1.5 \mathrm{~m} / \mathrm{s}$ and $0.7 \mathrm{~m} / \mathrm{s}$, respectively, for both $\mathrm{u}$ and $\mathrm{v}$ components. The contribution of the NWP local biases to the total error SD is estimated to be of about $1 \mathrm{~m} / \mathrm{s}$. The latter is estimated by analyzing the real local ASCAT-NWP differences for different 5-day periods in 2013 (as those of Fig. 1). As such, the random error SD contribution to the
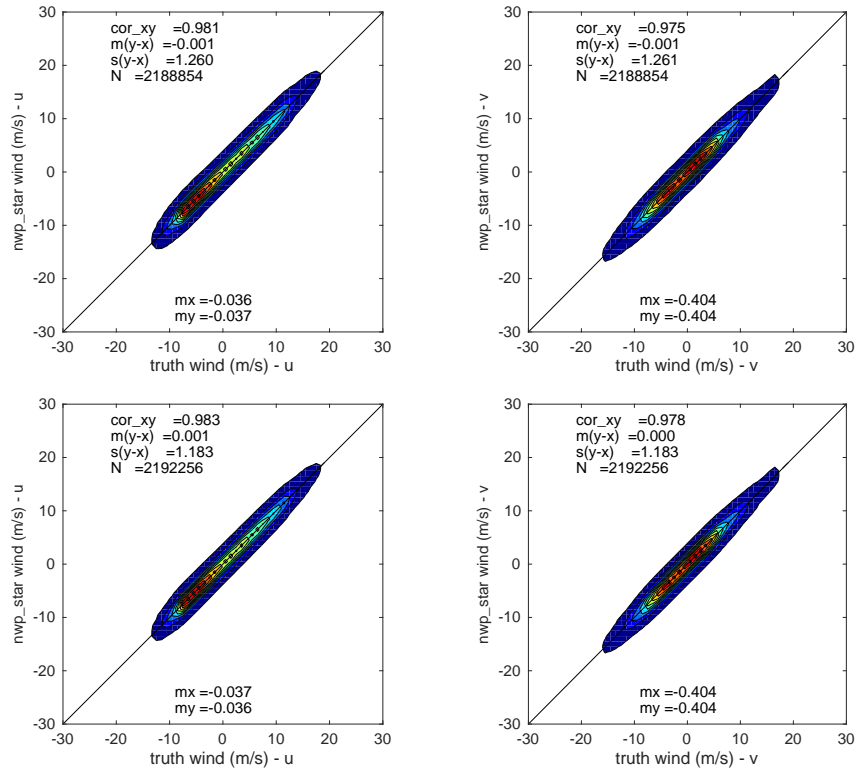

Fig. 2. Two-dimensional histogram of improved NWP forcing (nwp_star) vs. truth, zonal wind components (left) and meridional wind component $\mathrm{v}$ (right): The number of data is denoted by $\mathrm{N}$; $\mathrm{mx}$ and my are the mean values along the $\mathrm{x}$ and $\mathrm{y}$ axes, respectively; $\mathrm{m}(\mathrm{y}-\mathrm{x})$ and $\mathrm{s}(\mathrm{y}-\mathrm{x})$ are the bias and the standard deviation with respect to the diagonal, respectively; and cor $\mathrm{xy}$ is the correlation value between the $\mathrm{x}$ - and $\mathrm{y}$-axis distributions. Top panel, scatterometer correction applied to nwp winds contains data from ASCAT-A. Bottom panel, scatterometer correction contains data from both ASCAT-A and B. The ECMWF forecast at 3 UTC, on the central day of the scatterometer correction, is used as truth.

total NWP error is about $1.1 \mathrm{~m} / \mathrm{s}$. In summary, the following Gaussian perturbations from the true wind field are applied to generate the NWP SEW and the unbiased ASCAT winds: $N_{n w p}(1,1.1), N_{\text {ascat }}(0,0.7)$, respectively.

The simulated NWP SEW corrected field (hereafter referred to as NWP*) is then calculated as the sum of the simulated NWP SEW field and the scatterometer-based correction, which is applied at each grid point on the map. Note that the same procedure is applied to real data in Section 4.2.

\section{RESULTS}

\subsection{Simulation results}

Figure 2 illustrates the ability of the scatterometer correction to remove the NWP local biases, as well as the maximum noise reduction achieved globally with the defined settings (see previous section), on the $12^{\text {th }}$ to the $16^{\text {th }}$ January 2013. With an only ASCAT-A based correction, the NWP total error $\mathrm{SD}$ is reduced from $1.50 \mathrm{~m} / \mathrm{s}$ to about $1.26 \mathrm{~m} / \mathrm{s}$, while adding ASCAT-B sampling to the correction reduces the total error to 
$1.18 \mathrm{~m} / \mathrm{s}$. The ability to reduce the total error increases with latitude, along with the number of samples accumulated by location (not shown). Also note that the addition of ASCAT-B sampling results in a substantial NWP total error reduction in the tropics, while at mid and high latitudes the error reduction is marginal (not shown). This is because the scatterometer sampling is very limited in the tropics but by adding ASCAT$\mathrm{B}$ it is doubled.

With the current systematic and random error settings (see previous section), it is estimated that at least four measurements per grid point are required to reduce the total NWP error and therefore provide a more accurate ocean forcing. For the ASCAT-A only configuration, in about $12 \%$ of the global data ( $25.7 \%$ of tropical data), the impact of the correction is at best neutral (this result is consistent for several sets of 5 consecutive days within 2013). For combined sampling from ASCAT-A and B this value reduces to $0.66 \%$ globally.

\subsection{Case study: West African coast}

In this section, an example of a poorly sampled area, i.e., the West African coast, is analyzed. This area is mainly sampled 3 or 4 times (depending on the grid point location) every 5 days, although a very reduced fraction of the grid points are sampled less than 3 times. Figure 3 illustrates how moist convection induced wind variability may be present south of the West African coast. It is striking how this wind effect is much better captured by the corrected NWP SEW field (bottom) than by the original NWP SEW field (top), as suggested by the fine-scale structure of the former.

\section{CONCLUSIONS}

The Monte Carlo simulation is an effective tool to evaluate the impact of satellite sampling in the proposed scatterometerbased correction for an improved NWP SEW forcing. As expected, the skill of the correction depends on the number of passes, and thus on the latitude. It is beneficial to use a scatterometer-based correction when there are at least 4 scatterometer wind samples within the selected temporal window, while for 3 samples the impact is neutral, and below 3 the impact is detrimental. Less than $0.5 \%$ of the ocean surface is only sampled two times by ASCAT-A over the 5-day time window, and these grid points are all confined within the tropical band [-30S 30N]. However, the corrected NWP forcing field is mostly improved at high latitudes. When adding the ASCAT-B sampling, the corrected NWP wind errors substantially reduce in the tropics too. Finally, although the simulation indicates that the ASCAT-A only configuration is not optimal in the tropics, real data (qualitative) analysis show that the corrected NWP fields contain fine-scale structure as expected in areas of moist convection as is the case of the West African coast. A more comprehensive validation of the
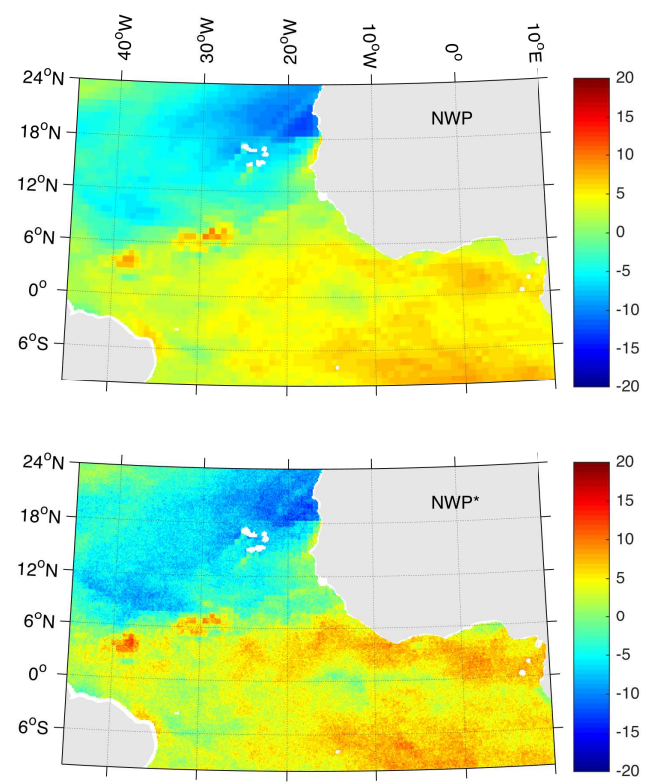

Fig. 3. Reginal map illustrates the meridional stress equivalent wind component encompassing the West African coast on 14th June, 2013. On the top panel, NWP (ECMWF) stress equivalent meridional component, at 03 UTC. Below the new wind forcing NWP*, with additional information from the scatterometer. The colors represent the magnitude in $\mathrm{m} / \mathrm{s}$ (see color scale).

scatterometer-based corrections will be carried out in the near future.

\section{ACKNOWLEDGMENTS}

A. Trindade is funded by an FPI grant (BES-2013-064521) from the Spanish Ministry of Economy and Competitiveness (MINECO) R\&D project MIDAS-7 (reference AYA201239356-C05-03). We thank Anton Verhoef for sharing the reprocessed ASCATs $12.5 \mathrm{~km} \mathrm{L2}$ wind data for 2013. 


\section{REFERENCES}

[1] S. Silvareddy, M. Ravichandran, and M. S. Girishkumar, "Evaluation of ascat-based daily gridded winds in the tropical indian ocean," Journal of Atmospheric and Oceanic Technology, vol. 30, no. 7, pp. 1371-1381, 2013.

[2] D. Chelton, M. Schlax, M. Freilich, and R. Milliff, "Satellite measurements reveal persistent small-scale features in ocean winds," Science, vol. 303, pp. 978-983, February 2004.

[3] A. Stoffelen, A. Verhoef, J. Vogelzang, J. de Kloe, M. Belmonte, and Verspeek J., "A long-term ocean wind and stress vector cdr," Presentation IOVWST.

[4] Jur Vogelzang, Ad Stoffelen, Anton Verhoef, and Julia Figa-Saldaña, "On the quality of high-resolution scatterometer winds," Journal of Geophysical Research: Oceans, vol. 116, no. C10, 2011, C10033. 